\title{
Emprego de resina epóxi em vigas danificadas de madeira de Pinus elliottii
}

\author{
Employment of epoxy resin in Pinus elliottii timber \\ damaged beams
}

\section{Marília da Siva Bertolini \\ Diego Henrique de Almeida \\ Laurenn Borges de Macedo \\ Felipe Hideyoshi Icimoto \\ Fabiane Salles Ferro \\ André Luis Christoforo \\ Francisco Antonio Rocco Lahr}

\section{Resumo}

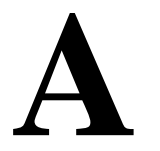

utilização da madeira em estruturas está vinculada a condições que permitam sua utilização por muitos anos sem a perda de suas propriedades de resistência e de rigidez. Entretanto, por se tratar de material natural, as estruturas em madeira projetadas podem estar sujeitas ao ataque de agentes biológicos, ação de intempéries, entre outros fatores, requerendo soluções na forma de reparo ou reforço. O presente trabalho objetivou investigar a influência do uso de resina epóxi como agente reparador em vigas danificadas de madeira de Pinus elliottii no cálculo do produto de rigidez à flexão. Para tanto, foi realizado um planejamento experimental completo, tendo a posição dos defeitos provocados (central ou laterais), o uso ou não da resina nos defeitos, e a posição desses defeitos (superior - compressão ou Inferior - tração) como fatores. Os resultados da análise estatística revelaram que o uso da resina epóxi nas regiões danificadas das vigas apresentaram valores do produto de rigidez na flexão significativamente superiores quando comparados com as vigas da condição com defeito e sem a resina epóxi. Mesmo sendo significativo o uso da resina nas

Marília da Silva Bertolini Universidade de São Paulo São Carlos - SP - Brasil

Diego Henrique de Almeida Pontifícia Universidade Católica de Minas Gerais

Poços de Caldas - MG - Brasil

Laurenn Borges de Macedo Universidade de São Paulo São Carlos - SP - Brasil

Felipe Hideyoshi Icimoto Universidade de São Paulo São Carlos - SP - Brasil

Fabiane Salles Ferro Universidade de São Paulo São Carlos - SP - Brasil

André Luis Christoforo Universidade Federal de São Carlos São Carlos - SP - Brasil

Francisco Antonio Rocco Lahr Universidade de São Paulo São Carlos - SP - Brasil

Recebido em 17/06/13 Aceito em 17/06/14

regiões danificadas, o produto de rigidez à flexão das vigas reparadas foi inferior ao produto de rigidez à flexão das vigas sem defeito (referência), evidenciando a necessidade de outros estudos com outras resinas e outros materiais visando à recuperação total da rigidez da peça de madeira danificada.

Palavras-chave: Madeira. Vigas. Resina epóxi. Reforço estrutural.

\section{Abstract}

The use of wood in structures is linked to conditions that allow its use for many years without losing its strength and stiffness properties. However, since it is a natural material, wood engineered structures may be subject to attack by

biological agents, and weathering action, among other factors, requiring solutions for repair or reinforcement. The aim of this research study is to investigate the influence of using epoxy resin as a healing agent for damaged Pinus elliottii wooden beams in the calculation of bending stiffness for the product. Therefore, a comprehensive experiment was performed, considering the position of caused defects (central and sides), the use or not of resin in the damaged regions, and the position of these damages (superior - compression or inferior - tensile) as factors. The results of the statistical analysis revealed that the use of epoxy resin in the beam damaged regions provided a bending stiffness significantly higher when compared to damaged beams without the epoxy resin. Despite being significant the use of the resin in the damaged regions, the bending inertia of the repaired beams was smaller compared to the beams without damage (reference), pointing out the need for further studies with other resins and materials in order to obtain full recovery of the stiffness in the damaged wood.

Keywords: Wood. Beams. Epoxy resin. Structural reinforcement.
\end{abstract}




\section{Introdução}

A utilização da madeira como material estrutural requer cuidados para minimizar os problemas referentes à diminuição das propriedades de resistência e de rigidez da madeira com o passar dos anos.

Para a realização de projetos de estruturas de madeira, a Associação Brasileira de Normas Técnicas (ABNT) fornece em seu documento normativo NBR 7190 (ABNT, 1997) as diretrizes para um dimensionamento correto, levando em consideração as propriedades físicas e mecânicas da madeira empregada, porém os problemas que podem ocorrer em elementos estruturais de madeira empregados em estruturas perenes são vários.

As propriedades de resistência e de rigidez da madeira são diretamente influenciadas por seu teor de umidade. Para efeito de comparação entre resistências da madeira provenientes de espécies diferentes, é necessária a adoção de um teor de umidade padrão, sendo adotado pela norma brasileira o teor de umidade igual a $12 \%$. O acréscimo do teor de umidade é responsável por diminuir as propriedades de resistência da madeira (LOGSDON; CALIL JUNIOR, 2002; SILVEIRA; REZENDE; VALE, 2013)

Com o elevado teor de umidade, outros problemas podem ocorrer em estruturas de madeira que também minimizam a resistência dos elementos estruturais, causando defeitos. A madeira de uma estrutura exposta ao contato com água ou em ambientes muito úmidos se torna favorável ao aparecimento de organismos deterioradores da madeira, como, por exemplo, fungos, bactérias, cupins e brocas, entre outras patologias (PAES; MORAIS; LIMA, 2005; COSTA, 2009; RIDOUT, 2009; LEILA et al., 2012). Por isso, o prétratamento da madeira antes de sua utilização é importante, como relatam Bertolini et al. (2013), Calil Neto (2011), Vivian et al. (2012) e Silva et al. (2013)

Outra situação que pode proporcionar danos a uma estrutura de madeira é a ocorrência de incêndios, como pode ser observado nos trabalhos de Pinto (2005), Regobello et al. (2006), Figueroa e Moraes (2009) e Molina et al. (2012).

Muitos dos problemas encontrados em estruturas de madeira estão relacionados à falta de manutenções periódicas; muitas chegam ao estadolimite último e não podem ser mais utilizadas, a ponto de necessitar reparos de algumas partes ou até sua reconstrução (SARTORTI, 2008; MILANI; KRIPKA, 2012; CARVALHO et al., 2012).
Para a recuperação de uma estrutura de madeira devem ser identificadas três etapas básicas após um estudo cuidadoso e crítico da estrutura: informações sobre a estrutura existente; estimativa de sua capacidade de carga; e desenho do reforço juntamente com os detalhes de recuperação (BORGES; SÁLES, 2007).

Alguns métodos podem ser utilizados com a finalidade de recuperação de estruturas: substituição das peças comprometidas ou solidarização de elementos que completem a capacidade mecânica dos elementos estruturais danificados. A primeira alternativa é uma das técnicas mais utilizadas, porém encontra limitações, como a indisponibilidade de peças com mesmas dimensões, custos elevados e incompatibilidade das espécies atualmente disponíveis com as características físicas exigidas pela estrutura, principalmente quando se remete às estruturas históricas (NOWAK; JASIENKO; CZEPIZAK, 2013).

Dessa forma, estudos vêm sendo realizados sobrea utilização de fibras sintéticas e naturais com o intuito de reforço estrutural e na reabilitação de sistemas estruturais de madeira (FIORELLI; DIAS, 2003; MIOTTO; DIAS, 2011). Outra técnica utilizada que também se apresenta eficiente na reabilitação de estruturas consiste no uso de resina, assim como discutido no trabalho de Leila et al. (2012), entretanto são poucas as pesquisas que envolvem o emprego de materiais compósitoparticulados como reforço nas estruturas de madeira (BRAZ et al., 2013), o que motiva o desenvolvimento de novas pesquisas.

Nesse contexto, o presente estudo objetivou investigar, com o uso de metodologia de planejamento fatorial de experimentos, a influência do uso de resina epóxi no produto de rigidez à flexão em vigas danificadas de madeira de Pinus elliottii, para contribuir com informações na referida temática.

\section{Material e métodos}

Os fatores e níveis estipulados para verificação da eficiência do emprego da resina epóxi (bicomponente) na rigidez à flexão em corpos de prova de madeira de Pinus elliottii submetidos a esforços de flexão foram:

(a) a posição dos defeitos provocados (Região Central; Regiões Laterais), o uso ou não da resina nos defeitos (sem; com); e

(b) a localização desses defeitos (Superior região comprimida; Inferior - região tracionada).

122 Bertolini, M. da S.; Almeida, D. H. de; Macedo, L. B. de; Icimoto, F. H.; Ferro, F. S.; Christoforo, A. L.; Lahr, F. A. R. 
A combinação dos níveis dos fatores estipulados forneceu um planejamento experimental completo do tipo $2^{3}$, dotado de oito condições experimentais distintas, explicitadas na Tabela 1 e ilustradas na Figura 1.

Cabe ressaltar que a condição de referência (Ref) ou testemunha não faz parte do planejamento experimental, sendo os valores médios dos produtos de rigidez à flexão de referência comparados com os valores médios dos produtos de rigidez à flexão das oito condições experimentais investigadas.

A partir de um lote homogêneo de madeira de Pinus elliottii, com teor de umidade na ordem de $12 \%$, foram retirados 6 corpos de prova para realização do ensaio de flexão estática, respeitando a relação entre o comprimento $(L)$ e a altura da seção transversal $(h)$ estabelecida pela norma brasileira NBR 7190 (ABNT, 1997) $(L / h \geq 21)$.

Para simulação dos defeitos nos corposdeprova foram produzidas lacunas, simulando regiões deterioradas removidas, possuindo $8 \mathrm{~cm}$ de comprimento, $1,10 \mathrm{~cm}$ de profundidade (um quarto da medida da seção transversal do corpodeprova) e $5 \mathrm{~cm}$ de largura (mesma largura do corpodeprova). Essas lacunas foram feitas na região central do corpodeprova e posteriormente nas regiões laterais, igualmente espaçadas da lacuna localizada ao centro (Figura 4). Para a restauração, utilizou-se resina epóxi estrutural bicomponente com endurecedor em poliaminoamida. A resina e o endurecedor foram misturados na proporção em massa de uma parte de resina para meia de endurecedor de acordo com as especificações do fabricante. O tempo especificado para cura total da resina é de 7 dias, sendo esse período respeitado para a realização dos ensaios. A Figura 1 apresenta as lacunas nos corposdeprova e preenchidas com a resina epóxi.

O ensaio utilizado para a obtenção do produto de rigidez (Equação 1) foi o de flexão estática a quatro pontos (Figuras 2 e 3), seguindo a premissa de métodos de cálculo da norma americana D198 (AMERICAN..., 1997), adaptada para a condição de ensaio não destrutivo, em razão de o deslocamento no ponto médio da peça estar limitado à razão L/200, em que L é a distância entre apoios, medida que garante comportamento linear físico e geométrico para as peças de madeira testadas, sendo condição de pequenos deslocamentos requerida pela norma brasileira NBR 7190 (ABNT, 1997) para verificação do estado-limite de utilização (ELU). Ressalta-se que as 6 peças de madeira sem defeito foram ensaiadas inicialmente nas duas posições (Inferior; Superior), e, posteriormente, os defeitos no ponto médio foram provocados, e ensaiadas as vigas da condição sem e com o uso da resina epóxi e nas posições superior e inferior, terminando com a execução dos danos laterais e posterior dos ensaios nas condições sem e com a resina e nas duas posições distintas, fornecendo 8 ensaios por peça (Figura 4), totalizando 48 experimentações para o planejamento, mais 12 referentes à testemunha (peça sem defeito).

$$
E I=\frac{F \cdot a \cdot\left(3 \cdot L^{2}-4 \cdot a^{2}\right)}{48 \cdot \delta_{L / 200}}
$$

Eq. 1

Tabela 1 - Condições experimentais investigadas

\begin{tabular}{ccccccc}
\hline & \multicolumn{2}{c}{ Localização dos Defeitos } & \multicolumn{2}{c}{ Resina } & \multicolumn{2}{c}{ Posição dos Defeitos } \\
\hline Condição* & Central & Lateral & Sem & Com & Superior & Inferior \\
\hline RCsemSup & $\mathrm{X}$ & & $\mathrm{X}$ & & $\mathrm{X}$ & \\
RCsemInf & $\mathrm{X}$ & & $\mathrm{X}$ & & & $\mathrm{X}$ \\
RCcomSup & $\mathrm{X}$ & & & $\mathrm{X}$ & $\mathrm{X}$ & \\
RCcomInf & $\mathrm{X}$ & & & $\mathrm{X}$ & & $\mathrm{X}$ \\
RLsemSup & & $\mathrm{X}$ & $\mathrm{X}$ & & $\mathrm{X}$ & \\
RLsemInf & & $\mathrm{X}$ & $\mathrm{X}$ & & & $\mathrm{X}$ \\
RLcomSup & & $\mathrm{X}$ & & $\mathrm{X}$ & $\mathrm{X}$ & \\
RLcomInf & & $\mathrm{X}$ & & $\mathrm{X}$ & & $\mathrm{X}$ \\
\hline
\end{tabular}

Nota: Legenda:

${ }^{*} \mathrm{RC}=$ reforço central;

$\mathrm{RL}=$ reforço lateral;

sem = sem preenchimento dos defeitos pela resina;

com = com preenchimento dos defeitos pela resina;

Sup = defeito localizado na posição superior (região comprimida para ensaio); e

Inf = defeito localizado na posição inferior (região tracionada para ensaio). 
Figura 1 -Corpos de prova de flexão estática: (a) lacunas simulando os defeitos; (b) lacuna central preenchida com resina epóxi; e (c) lacunas central e laterais preenchidas com resina epóxi

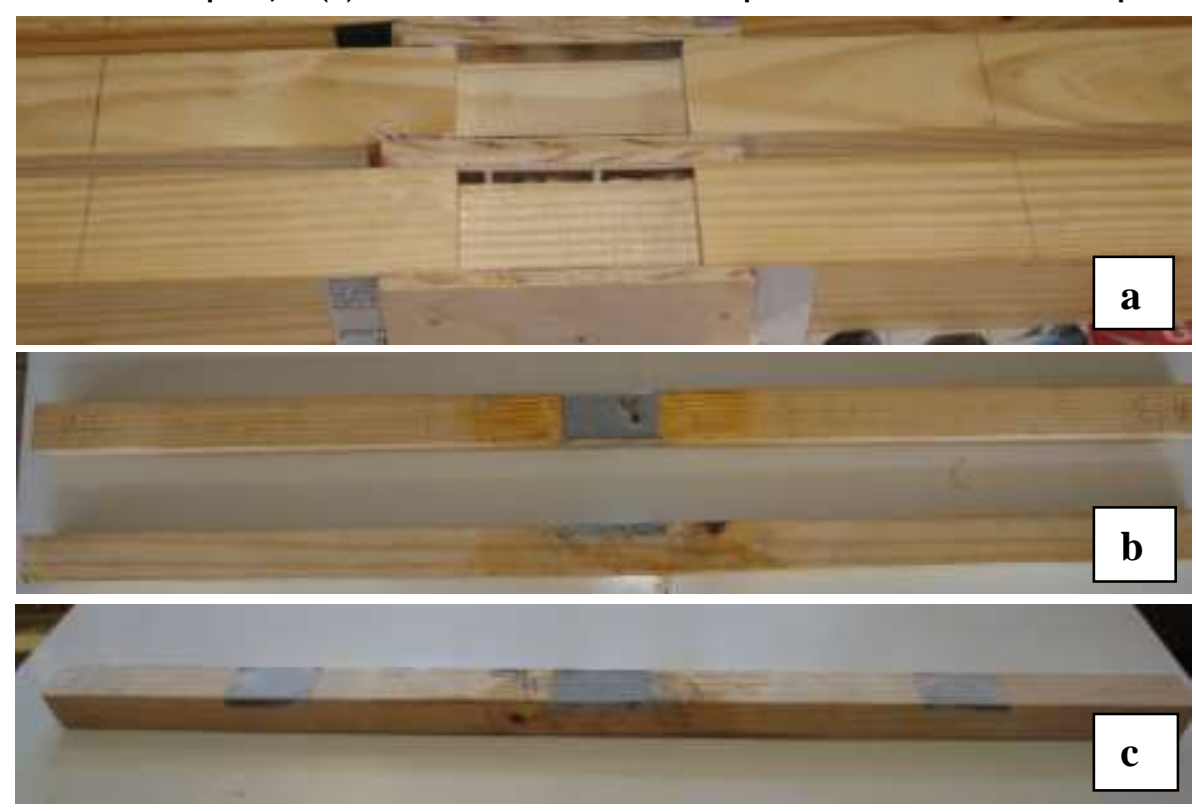

Figura 2 - Flexão estática a quatro pontos

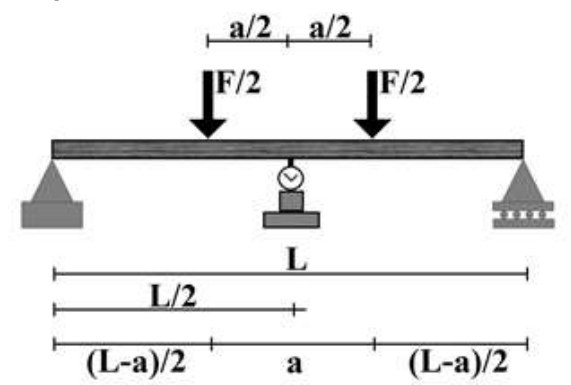

Figura 3 - Ensaio de flexão estática nas peças analisadas

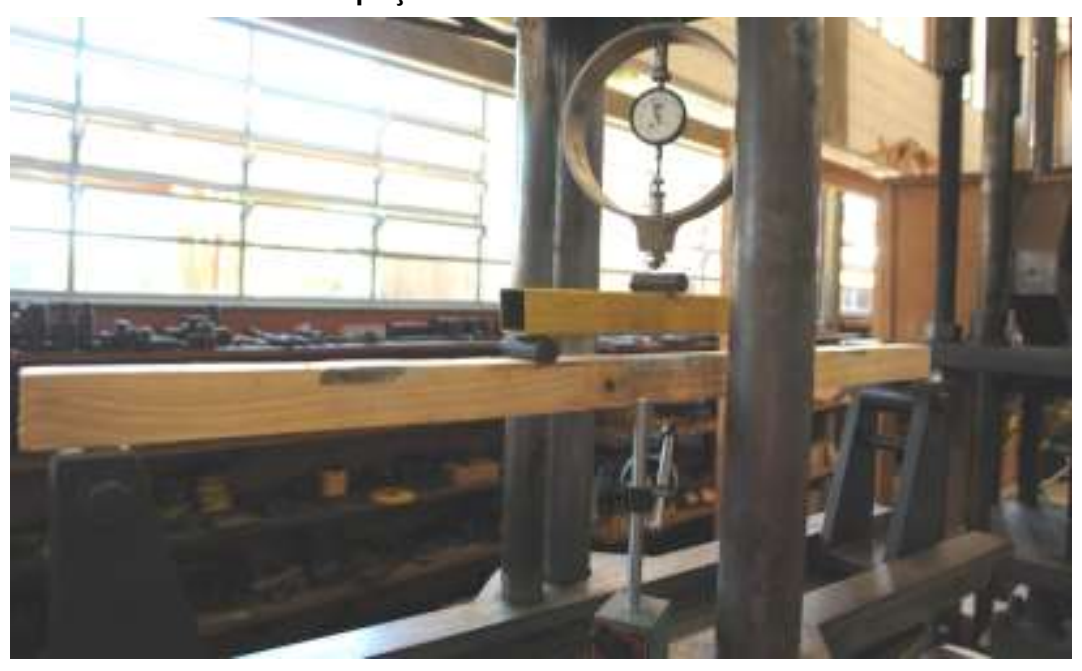

124 Bertolini, M. da S.; Almeida, D. H. de; Macedo, L. B. de; Icimoto, F. H.; Ferro, F. S.; Christoforo, A. L.; Lahr, F. A. 
Figura 4 - Ilustração dos fatores e níveis estipulados

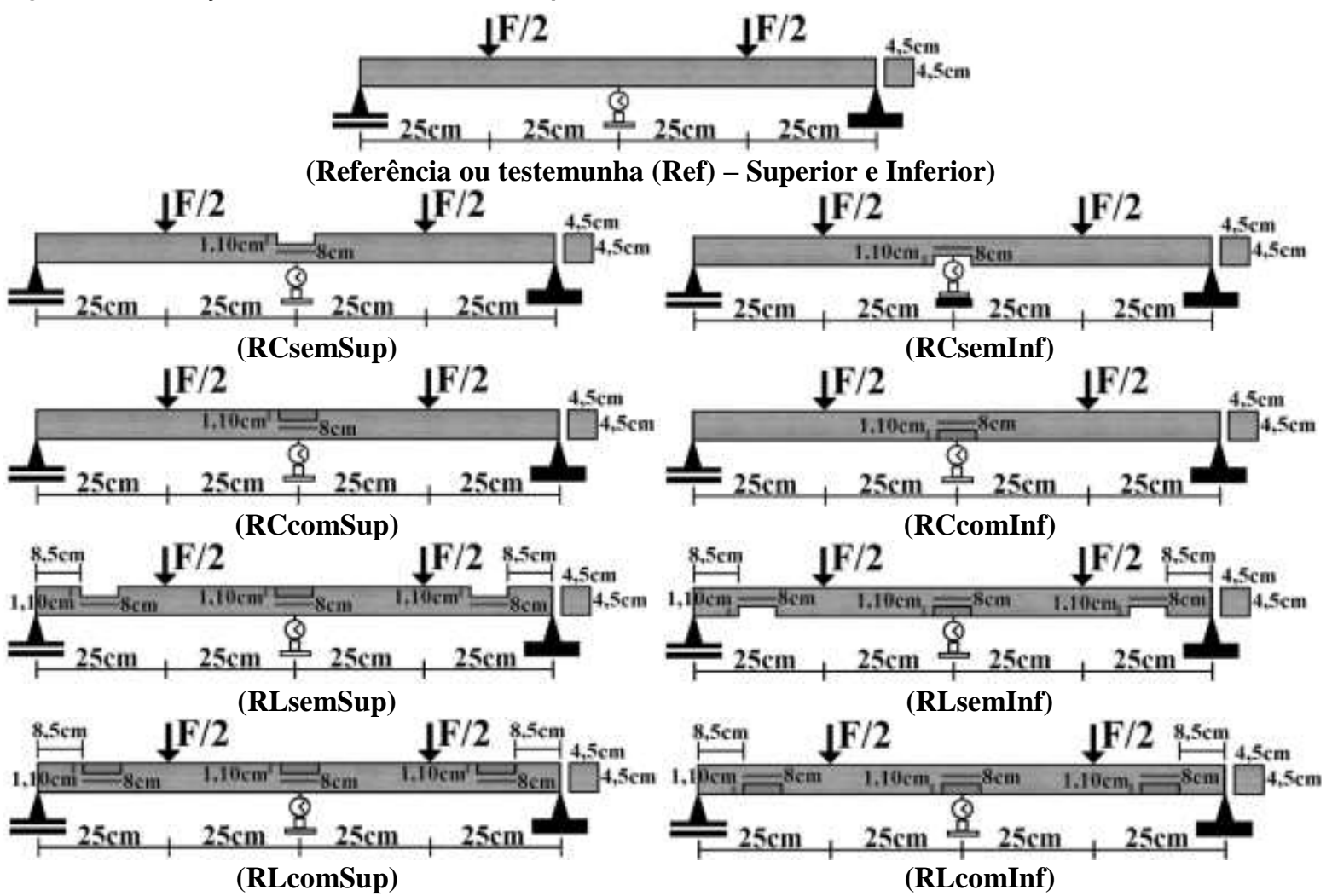

Da Equação 1, EI denota o produto de rigidez à flexão, $F$ é o valor da força aplicada responsável pelo deslocamento no meio do vão igual a $L / 200$ $\left(\delta_{\mathrm{L} / 200}\right)$, e $a$ é a distância entre os pontos de aplicação da carga.

Para a verificação da eficiência do emprego da resina epóxi nos corpos de prova danificados de madeira de Pinus elliottii foi utilizada a análise de variância (ANOVA), avaliada ao nível de 5\% de significância, tendo a equivalência entre médias para os produtos de rigidez à flexão como hipótese nula $\left(\mathrm{H}_{0}\right)$, e a não equivalência entre os produtos de rigidez à flexão como hipótese alternativa $\left(\mathrm{H}_{1}\right)$. P-valor superior ao nível de significância do teste implica aceitar $\mathrm{H}_{0}$; em caso contrário, é rejeitada.

Para validação da ANOVA, foram investigadas a normalidade na distribuição dos produtos de rigidez à flexão e a homogeneidade entre variâncias, com o auxílio dos testes de AndersonDarling e Bartlett e Levene, respectivamente, ambos ao nível de 5\% de significância.

Para o teste de Anderson-Darling, a hipótese nula consistiu em assumir normalidade na distribuição, e a não normalidade como hipótese alternativa. $\mathrm{P}$ valor superior a $5 \%$ implica aceitar $\mathrm{H}_{0}$, refutando-a em caso contrário. Os testes de Bartlett e Levene foram formulados considerando a equivalência entre as variâncias como hipótese nula, e a não

equivalência como hipótese alternativa. P-valor superior ao nível de significância implica aceitar $\mathrm{H}_{0}$, rejeitando-a em caso contrário.

\section{Resultados e discussão}

A Tabela 2 apresenta os resultados dos produtos de rigidez à flexão das madeiras de Pinus elliottii, sendo $\bar{x}$ a média amostral, $S d$ o desvio padrão, e $C v$ o coeficiente de variação.

Os resultados médios do produto de rigidez à flexão das peças sem defeito (referência) para a posição Superior (compressão na região do defeito) foram $36 \%, 29 \%, 29 \%$ e $16 \%$ superiores aos produtos de rigidez das peças das condições experimentais RCsemSup, RCcomSup, RLsemSup e RLcomSup respectivamente, e os resultados médios do produto de rigidez à flexão das peças sem defeito para a posição Inferior (tração na região do defeito) foram 36\%, 33\%, 35\% e $15 \%$ superiores aos produtos de rigidez à flexão das peças das condições experimentais RCsemInf, RCcomInf, RLsemInf e RLcomInf respectivamente, revelando não ser possível a recuperação total da rigidez à flexão com o uso da resina epóxi nas condições de rasgo (defeitos) investigadas.

A Tabela 3 apresenta os resultados da ANOVA (com 47 graus de liberdade) dos fatores $\mathrm{e}$ 
interações investigados sobre produto de rigidez à flexão (EI) juntamente com os resultados do teste de normalidade de Anderson-Darling e homogeneidade entre variâncias de Bartlett e Levene, estando sublinhados os P-valores considerados significativos pela ANOVA (Pvalor $<0,05)$.

Devido aos P-valores encontrados superiores ao nível de significância dos testes de AndersonDarling, Bartlett e Levene, constata-se serem normais as distribuições e equivalentes as variâncias entre as condições experimentais investigadas, validando o modelo de ANOVA. Ressalta-se que os resíduos da análise de variância também foram verificados e atendidos em todos os casos, sendo utilizado o teste de Anderson-Darling para avaliação da normalidade e os gráficos de resíduos versus ordem de observação e resíduos versus valores ajustados para avaliação da independência e homogeneidade respectivamente.

Da Tabela 3, os fatores considerados significativos pela ANOVA sobre o produto de rigidez à flexão foram o defeito (Central; Laterais) e o reforço (com; sem), não sendo significativa a influência do fator Posição (Superior; Inferior), nem das interações dos fatores investigados. A Tabela 4 apresenta os resultados do teste de Tukey dos fatores considerados significativos pela ANOVA. Letras iguais implicam tratamentos com médias equivalentes.

Tabela 2 - Produtos de rigidez à flexão por condição experimental investigada

\begin{tabular}{cccccc}
\hline \multicolumn{5}{c}{ EI $\left(\mathbf{k N} \cdot \mathbf{m}^{2}\right)$} \\
\hline Estat. & RefSup & RefInf & RCsemSup & RCsemInf & RCcomSup \\
\hline $\bar{x}$ & 4,53 & 4,49 & 3,32 & 3,30 & 3,52 \\
$S d$ & 0,55 & 0,55 & 0,29 & 0,28 & 0,39 \\
$C v(\%)$ & 12 & 12 & 9 & 9 & 11 \\
\hline \multicolumn{5}{c}{ EI $\left(\mathbf{k N} \cdot \mathbf{m}^{2}\right)$} \\
\hline Estat. & RCcomInf & RLsemSup & RLsemInf & RLcomSup & RLcomInf \\
\hline $\bar{x}$ & 3,37 & 3,50 & 3,33 & 3,94 & 3,92 \\
$S d$ & 0,36 & 0,38 & 0,34 & 0,32 & 0,23 \\
$C v(\%)$ & 11 & 11 & 10 & 8 & 6 \\
\hline
\end{tabular}

Nota: Legenda:

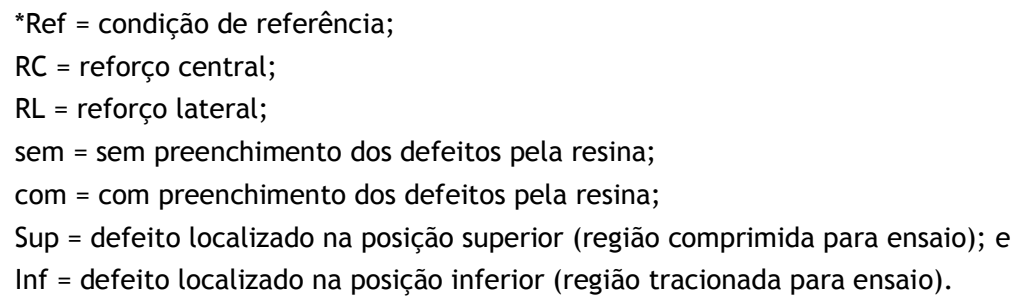

Tabela 3 - Resultados da ANOVA e dos testes de normalidade e equivalência entre variâncias para o produto de rigidez à flexão

\begin{tabular}{ccccc}
\hline & \multicolumn{5}{c}{ P-valor } \\
\hline Fatores e Interações & EI (ANOVA) & Anderson-Darling & Bartlett & Levene \\
\hline Defeito (D) & $\underline{0,011}$ & & & \\
Reforço (R) & $\underline{0,005}$ & & & \\
Posição (P) & 0,442 & 0,207 & 0,503 & 0,829 \\
$\mathrm{D} \times \mathrm{R}$ & 0,065 & & & \\
$\mathrm{D} \times \mathrm{P}$ & 0,969 & & \\
$\mathrm{R} \times \mathrm{P}$ & 0,935 & & \\
$\mathrm{D} \times \mathrm{R} \times \mathrm{P}$ & 0,457 & & & \\
\hline
\end{tabular}

Tabela 4 - Resultados do teste de Tukey

\begin{tabular}{ccccc}
\hline & \multicolumn{2}{c}{ Defeito } & \multicolumn{2}{c}{ Reforço } \\
\hline & Central & Laterais & Sem & Com \\
\hline $\bar{x}\left(\mathrm{kN} \cdot \mathrm{m}^{2}\right)$ & 3,39 & 3,67 & 3,37 & 3,68 \\
Agrupamento & B & A & B & A \\
\hline
\end{tabular}

126 Bertolini, M. da S.; Almeida, D. H. de; Macedo, L. B. de; Icimoto, F. H.; Ferro, F. S.; Christoforo, A. L.; Lahr, F. A. R. 
Os resultados da Tabela 4 revelaram que a inclusão da resina forneceu valores do produto de rigidez à flexão superiores aos valores da condição sem o reforço (Central e Laterais), revelando ser significativo o uso da resina nas vigas de madeira reparadas. Pode-se relacionar esse comportamento com uma compatibilidade satisfatória da resina com a madeira, por meio de um bom nível de ancoragem. O produto de rigidez à flexão nas regiões laterais dos corpos de prova com resina foi superior ao produto de rigidez à flexão na região central dos corpos de prova com resina, em razão dos menores níveis da tensão normal de compressão (que provocam menores níveis de deformação) quando comparado com os níveis de tensão normal de compressão na região central, justificados pelos maiores valores do momento fletor na parte central da peça. $O$ fato de já existir o defeito central nos corpos de prova pode ter influenciado nos valores de rigidez à flexão obtidos quando do posterior emprego da resina nos defeitos localizados nas extremidades. Entretanto, esse fator foi considerado fixo (com exceção da condição de referência), não sendo investigada nesta pesquisa tal influência, o que decorre do planejamento do experimento delineado.

Estudos análogos obtiveram excelente desempenho para emendas com resina epóxi, como Cruz e Mendonça (2002), Custódio, Broughton e Cruz (2011), Pizzo, Gavioli e Lauriola (2013), entre outros, ressaltando-se a eficácia desse tipo de restauração. No entanto, Cruz e Mendonça obtiveram redução de $65 \%$ no módulo de elasticidade longitudinal na flexão estática para peças de madeira restauradas, com emendas geradas no centro do corpo de prova, contendo resina epóxi e reforço de fibra de vidro. Os autores sugeriram estudos mais aprofundados em relação à adesão entre resina e madeira, pois, além da baixa rigidez, as peças se romperam justamente nessas regiões de contato.

Apesar de não ter sido avaliada a resistência à ruptura no trabalho em questão, os resultados superiores de rigidez sugerem boa compatibilidade entre resina e madeira em função também da espécie utilizada (Pinus elliottii), de maior permeabilidade. Além da espécie madeireira, as variáveis relacionadas à resina epóxi, como condições ambientais (umidade), condições de preparação e cura, têm efeito significativo no desempenho das restaurações, conforme demonstrado no estudo de Custódio, Broughton e Cruz (2011).

\section{Conclusões}

O uso da resina epóxi para restauração em peças danificadas de madeira de Pinus elliottii apresentou aumento significativo no produto de rigidez à flexão quando comparado à condição das vigas com defeito e sem o uso da resina, não sendo significativa a posição do reforço (Superior região comprimida; Inferior - região tracionada) sobre essa propriedade, evidenciando, assim, o desempenho considerável da resina. Ressalta-se também o desempenho das peças restauradas com epóxi mesmo quando solicitadas por tensões de tração, que poderiam comprometer a eficiência do uso dessa resina pela possível perda de aderência com a madeira (descolamento por tração).

Os valores de produto de rigidez obtido para vigas de madeira de Pinus elliottii com a restauração utilizando resina epóxi apresentou-se inferior àqueles das vigas sem defeito (referência). Estudos futuros com o emprego de fibras com a resina poderão ser realizados de modo a reforçar o desempenho, todavia neste trabalho ficou evidenciada a compatibilidade entre a madeira e a resina epóxi, além da viabilidade de restauração de peças no próprio local de uso dessas peças estruturais.

\section{Referências}

\section{AMERICAN SOCIETY FOR TESTING AND \\ MATERIALS. ASTM D-198: standard test method of static tests of lumber in structural sizes.West Conshohocken, Philadelphia, 1997.}

\section{ASSOCIAÇÃO BRASILEIRA DE NORMAS} TÉCNICAS. NBR 7190:projeto de estruturas de madeira. Rio de Janeiro, 1997.

BERTOLINI, M. S. et al.Accelerated Artificial Aging of Particleboards FromResidues of CCB Treated Pinus sp. and Castor Oil Resin. Materials Research, São Carlos, v. 16, n. 2, p. 293-303, 2013.

BORGES, M. L.; SÁLES, J. J. Recuperação Estrutural de Edificações Históricas Utilizando Perfis Formados a Frio. Cadernos de Engenharia de Estruturas, v. 39, p. 45-62, 2007.

BRAZ, J. C. F. et al. Numerical Evaluation of the Mechanical Behavior of a Particulate Composite Material as Reinforcement in Timber Beams. International Journal of Composite Materials, v. 3, p. 83-91, 2013. 
CALIL NETO, C. Madeira Laminada Colada (MLC): controle de qualidade em combinações espécie-adesivo-tratamento preservativo. São Carlos, 2011. 120 f. Dissertação (Mestrado em Ciência e Engenharia de Materiais) - Escola de Engenharia de São Carlos, Universidade de São Paulo, São Carlos, 2011.

CARVALHO, S. S. et al. Experimental Evaluation of the Employment of a Laminated Composite material with sisal fibers as reinforcement in timber beams. International Journal of Composite Material, v. 2, p. 97-100, 2012.

COSTA, M. A. Avaliação de Metodologias Alternativas Para Caracterização do ataque de Fungos Apodrecedores de Madeira. Brasília, DF, 2009. 83 f. Dissertação (Mestrado em Engenharia Florestal) - Escola de Engenharia, Universidade de Brasília, Brasília, 2009.

CRUZ, I. P.; MENDONÇA, M. Comportamiento de las Ligações Madeira-Resinas Sintéticas e Fibra de Vidrio na Restauração de Peças Estruturais. Tecnologia y Construccion, v. 18, n. 1, p. 37-43, 2002.

CUSTÓDIO, J.; BROUGHTON, J.; CRUZ, H. Rehabilitation of Timber Structures: preparation and environmental service condition effects on the bulk performance of epoxy adhesives.Construction and Building Materials, v. 25, p. 3570-3582, 2011.

FIGUEROA, M. J. M.; MORAES, P. D. Comportamento da Madeira a Temperaturas Elevadas. AmbienteConstruído, Porto Alegre, v. 9, n. 4, p. 157-174, out./dez. 2009.

FIORELLI, J.; DIAS, A. A. Analysis of the Strength and Stiffness of Timber Beams Reinforced With Carbon Fiber and Glass Fiber.Materials Research, São Carlos, v. 6, p. 193-202, 2003.

LEILA, F. M. et al. Avaliação do Emprego de Um compósito Particulado Como Reparo em Colunas de Madeira da Espécie Eucalyptus grandis. In: CONGRESSO NACIONAL DE ENGENHARIA MECÂNICA E INDUSTRIAL, 12.; CONGRESSO DE ENGENHARIAS DA UNIVERSIDADE FEDERAL DE SÃO JOÃO DEL-REI, 2., São João delRei, 2012. Anais...são João del Rei, 2012.

LOGSDON, N. B.; CALIL JUNIOR, C. Influência da Umidade nas Propriedades de Resistência e Rigidez da Madeira. Cadernos de Engenharia de Estruturas, n. 18, p. 77-107, 2002.
MILANI, C. J.; KRIPKA, M. A Identificação de Patologias em Pontes de Madeira: diagnóstico realizado no sistema viário do município de Pato Branco - Paraná. Revista Eletrônica de Engenharia Civil, v. 4, n. 1, p. 23-33, 2012.

MIOTTO, J. L.; DIAS, A. A. Avaliação Experimental de Vigas Mistas de MLC-Concreto Reforçadas Com Fibras de Vidro. Mecânica Experimental,v. 19, p. 121-131, 2011.

MOLINA, J. C. et al.Análise Numérica do Comportamento de Elementos de Madeira em Situações de Incêndio. Floresta e Ambiente, v. 19, n. 2, p. 162-170, 2012.

NOWAK, T. P.; JASIENKO, J.; CZEPIZAK, D. Experimental Tests and Numerical Analysis of Historic Bent Timber Elements Reinforced With CFRP Strips.Construction and Buildings Materials, v. 40, p. 197-206, 2013.

PAES, J. B.; MORAIS, V. M.; LIMA, C. R. Resistência Natural de Nove Madeiras do Semiárido Brasileiro a Fungos Causadores de Podridão Mole. Revista Árvore, v. 29, n. 3, p. 365-371, 2005.

PINTO, E. M. Determinação de Um Modelo de Taxa de Carbonização Transversal à Grã Para o Eucalyptus citriodora e Eucalyptus grandis. São Carlos, 2005. 119 p. Tese (Doutorado em Ciência e Engenharia de Materiais) - Escola de Engenharia de São Carlos, Universidade de São Paulo, São Carlos, 2005.

PIZZO, B.; GAVIOLI, M.; LAURIOLA, M. P. Evaluation of a Design Approach to the On-Site Structural Repair of Decayed Old Timber end Beams. Engineering Structures, v. 48, p. 611622, 2013.

REGOBELLO, R. et al. Análise Numérica e Experimental da Evolução de Temperatura na Madeira de Eucalyptus Exposta ao Fogo. In: ENCONTRO BRASILEIRO EM MADEIRA E EM ESTRUTURAS DE MADEIRA, 10., São Pedro, SP. Anais... São Pedro, SP, 2006.

RIDOUT, B. Timber Decay in Buildings: the conservation approach to treatment. London: English Heritage, 2009.

SARTORTI, A. L. Identificação de Patologias em Pontes de Vias Urbanas e Rurais no Munícipio de Campinas-SP. Campinas, 2008. 240 f. Dissertação (Mestrado em Engenharia Civil) - Faculdade de Engenharia Civil, Arquitetura e Urbanismo, Universidade Estadual de Campinas, Campinas, 2008. 
SILVA, M. R. et al.Strength and Stiffness of Thermally Rectified Eucalyptus Wood UnderComprression. Materials Research, v. 16, n. 5, p. 1077-1083, 2013.

SILVEIRA, L. H. C.; REZENDE, A. V.; VALE, A. T. Teor de Umidade e Densidade Básica da Madeira de Nove Espécies Comerciais

Amazônicas. Acta Amazônica, v. 43, n. 2, p. 179$184,2013$.
VIVIAN, M. A. et al.Qualidade doTratamento Preservativo em Autoclave Para a Madeira de Eucalyptus grandis e Eucalyptus cloeziana.

Scientia Forestalis, v. 40, n. 96, p. 445-453, 2012.

Marília da Silva Bertolini

Escola de Engenharia de São Carlos | Universidade de São Paulo | Av. Trabalhador Sãocarlense, 400, Centro | São Carlos - SP - Brasil | CEP 13566-590 | Tel.: (016) 3373-8206 | E-mail.: marília.bertolini@usp.br

\section{Diego Henrique de Almeida}

Pontifícia Universidade Católica de Minas Gerais | Departamento de Engenharia Civil | Av. Padre Francis Cletus Cox, 1661, Cowtry Club | Poços de Caldas - MG - Brasil | CEP 37701-355 | Tel.: (35) 3729-9200 | E-mail.: almeida@pucpcaldas.com.br

\section{Laurenn Borges de Macedo}

Escola de Engenharia de São Carlos | Universidade de São Paulo | E-mail.: laurennmacedo@hotmail.com

\section{Felipe Hideyoshi Icimoto}

Escola de Engenharia de São Carlos | Universidade de São Paulo | E-mail.: icimoto@usp.br

\section{Fabiane Salles Ferro}

Escola de Engenharia de São Carlos | Universidade de São Paulo | E-mail.: fsferro@usp.br

\section{André Luis Christoforo}

Departamento de Engenharia Civil | Universidade Federal de São Carlos | Rodovia Washington Luís, km 235, SP-310 | São Carlos - SP Brasil | CEP 36307-352 | Tel.: (16) 98259-6211 | E-mail.: alchristoforo@yahoo.com.br

\section{Francisco Antonio Rocco Lahr}

Escola de Engenharia de São Carlos | Universidade de São Paulo | E-mail.: frocco@sc.usp.br

\section{Revista Ambiente Construído}

Associação Nacional de Tecnologia do Ambiente Construído

Av. Osvaldo Aranha, $99-3^{\circ}$ andar, Centro

Porto Alegre - RS - Brasil

CEP 90035-190

Telefone: +55 (51) 3308-4084

Fax: +55 (51) 3308-4054

www.seer.ufrgs.br/ambienteconstruido

E-mail: ambienteconstruido@ufrgs.br 